\title{
(2)

\section{A APLICAÇÃO DA ABORDAGEM QUICK RESPONSE MANUFACTURING (QRM) NO PROCESSO DE DESENVOLVIMENTO DE NOVOS LÁPIS DE OLHOS}

\section{APPLICATION OF THE APPROACH QUICK RESPONSE MANUFACTURING (QRM) IN DEVELOPMENT PROCESS OF NEW EYE PENCILS}

\author{
Leandro Gomes Oliveira* E-mail: leandrobaquim@yahoo.com.br \\ Denise Cervilha de Freitas* E-mail: decervilha@gmail.com \\ Moacir Godinho Filho*E-mail: moacir@dep.ufscar.br \\ *Universidade Federal de São Carlos (UFSCar), São Carlos, SP
}

\begin{abstract}
Resumo: O Quick Response Manufacturing (QRM) é uma importante ferramenta para redução de lead time em todas as operações de uma empresa de manufatura. Embora existam trabalhos empíricos mostrando a aplicação do QRM no chão de fábrica, poucos trabalhos tratam de operações de escritório. Dentre estas operações, o desenvolvimento de produtos é uma área ainda muito pouco explorada. Este trabalho busca trazer contribuições para a literatura ao mostrar a possibilidade de implantação do QRM no processo de desenvolvimento de novos lápis de olhos em uma empresa fabricante de cosméticos. Após estudo de caso foi possível apresentar três propostas, viáveis economicamente, que se implantadas conjuntamente, poderá reduzir $55 \%$ do lead time desse processo. As propostas mostram também a importância de se repensar novas formas de se realizar o trabalho em escritório.
\end{abstract}

Palavras-chave: Quick Response Manufacturing. Operações de Escritório. Processo de Desenvolvimento de Produto. Cosméticos. Lápis de Olho.

\begin{abstract}
Quick Response Manufacturing (QRM) is an important tool for reducing lead time in all operations of a manufacturing company. Although there are empirical studies showing QRM application on the shop floor, few studies deal with office operations. Among these operations, product development is still a very little explored area. This paper aims to present contributions to the literature, showing the possibility of QRM application in the process of new eye pencil development at cosmetic company. In case study, it was possible to present three proposals, economically viable, if implemented together, will be able reduce $55 \%$ of lead time process. Proposals also show the importance of rethinking new ways of completing a job in office operations.
\end{abstract}

Keywords: Quick Response Manufacturing. Office Operations. Product Development Process. Cosmetics. Eye pencil.

\section{INTRODUÇÃO}

A velocidade tem ganhado força como fator competitivo, possibilitando rápida resposta às demandas dos clientes (SAPKAUSKIENE; LEITONIENE, 2010). O Quick Response Manufacturing (QRM) é uma abordagem que foca na busca implacável da 
redução de lead time ao longo de todas as operações para melhorar a qualidade, reduzir os custos e eliminar os desperdícios que não agregam valor. As empresas que implementam com sucesso o QRM tornam-se competidores formidáveis nos seus mercados, entregando produtos mais rápido que seus concorrentes (SOULÉ et al. 2016).

Apesar do foco na manufatura, as operações de escritório são responsáveis por uma fração considerável do lead time total (VEERAMANI; AGRAWAL, 1999). Além disso, influenciam a taxa de captação de pedidos, impactam na participação de mercado e são responsáveis por mais de $25 \%$ dos custos da mercadoria vendida (SURI, 2010). Segundo Suri (1998), existem quatro tarefas de operações de escritório importantes que afetam consideravelmente o lead time total: pedido de cotação, captura do pedido, processamento e processo de desenvolvimento do produto.

O Processo de Desenvolvimento de Produtos (PDP) é considerado um processo de negócio muito importante para a competitividade das empresas (MAGANHA et al. 2014). Sua eficácia pode ser tão importante para a sobrevivência de longo prazo quanto os próprios produtos desenvolvidos (LIZARELLI; TOLEDO, 2016). A cadeia da moda lida com nichos específicos que valorizam tendências estéticas, tornando esse mercado muito dinâmico. Dessa forma, cada vez mais se tem exigido a introdução de novos produtos com maior eficiência.

A Indústria Brasileira de Higiene Pessoal, Perfumaria e Cosméticos apresenta grande importância socioeconômica para o Brasil. Segundo a ABIHPEC (2016), essa indústria teve um crescimento no faturamento de $R \$ 4,9$ bilhões em 1996 para $\mathrm{R} \$ 42,6$ bilhões em 2015 e os empregos estão em grande desenvolvimento, sendo que as principais oportunidades na indústria, franquias, salões de beleza e consultoria de venda direta. As maquiagens ocupam a quinta posição de produtos mais consumidos no mundo, e uma que merece destaque é o lápis de olho, por apresentar grande volume de venda e ser um produto de baixo custo. No entanto, 0 desenvolvimento de novos lápis é um processo demorado e empresas que lançarem esses produtos mais rapidamente no mercado conseguirão maior market share e consequentemente, melhores retornos financeiros. 
Assim, o objetivo desse artigo é propor a aplicação da abordagem QRM na redução de lead time no processo de desenvolvimento de novos lápis para os olhos em uma empresa fabricante de cosméticos. Para isso, foi realizada uma revisão de literatura para dar embasamento teórico ao estudo de caso realizado. Com isso, pretende-se contribuir para a literatura relacionada ao QRM, que carece de trabalhos empíricos voltados a escritório e desenvolvimento de novos produtos (GODINHO FILHO; SAES, 2013).

Além dessa seção introdutória, o artigo está organizado em quatro seções. A segunda seção aborda a revisão da literatura, compreendendo o mercado nacional de cosméticos, as teorias de Processo de Desenvolvimento de Produto (PDP), Quick Response Manufacturing (QRM) e QRM em operações de escritório. A terceira seção demonstra o método de pesquisa e a quarta apresenta a empresa estudada e a partir disso, propostas de melhoria são apresentadas e os resultados analisados. Em seguida, na quinta seção, são explanadas as considerações finais do estudo.

\section{REFERENCIAL TEÓRICO}

A teoria para o desenvolvimento deste estudo está fundamentada nas temáticas de Processo de Desenvolvimento de Produto (PDP), Quick Response Manufacturing (QRM) e o QRM em operações de escritório.

\subsection{Processo de Desenvolvimento de Produto (PDP)}

O PDP é uma área de pesquisa amplamente abordada na literatura (TYAGI et al., 2013, VERMA et al., 2014). Tendo em vista a internacionalização dos mercados, o aumento da diversidade e variedade de produtos, a redução de ciclo de vida (MAGANHA et al. 2014), as crescentes exigências dos consumidores e prazos de lançamento (TOLEDO et al. 2008), o PDP é considerado um processo crítico para a obtenção de vantagem competitiva, que pode criar diferenciação, garantir barreiras à entrada, abrir novos mercados e aumentar receita e lucro das empresas (BERNARDI SOUZA; MORAES, 2016). 
O PDP situa-se na interface entre a empresa e o mercado, identificando as necessidades atuais e futuras de seus clientes. Ele pode ser compreendido como a sequência de atividades que a empresa utiliza para planejar, produzir e comercializar um novo produto ou aperfeiçoar um existente (LIZARELLI; TOLEDO, 2016). O desenvolvimento das atividades relacionadas ao PDP é um processo complexo que necessita da integração das áreas funcionais da empresa, principalmente Engenharia, P\&D, Marketing e Produção (JUGEND et al. 2010).

O desenvolvimento de novos produtos é um processo de tomada de decisão iterativo composto por estágios e filtros. Em uma visão mais abrangente, PDP é formado por etapas, que são constituídas por atividades, podendo ser desenvolvidas simultaneamente ou sequenciadas. A entrada em cada estágio é um ponto de decisão, que controla o processo e atua como garantia de qualidade, compondo um modelo denominado stage-gate (NORONHA et al. 2014). Com o crescimento da demanda por produtos inovadores, não apenas o número de atividades em cada estágio aumenta, mas também as relações entre elas tornam-se mais complexas (BERNARDI SOUZA; MORAES, 2016).

A abordagem utilizada para conduzir o PDP é influenciada principalmente pelo nível de maturidade da empresa (ECHEVESTE et al. 2016). Ele é um estágio evolutivo, composto por planos e ações bem delineados, que orientam as empresas para o diagnóstico e melhoria de desempenho. Além disso, ele também é um indicativo da sofisticação e da estabilidade com que práticas, técnicas e procedimentos são adotados (MAGANHA et al. 2014). Segundo Jugend et al. (2010), os níveis de maturidade podem ser divididos em: inicial, gerenciado, definido, quantitativamente gerenciado e em otimização (Quadro 1).

Por fim, o sucesso de um produto em um mercado competitivo requer que 0 PDP busque melhorias em vantagens competitivas em qualidade, custo e o tempo de entrega (lead time) (MENDES; TOLEDO, 2012; TYAGI et al. 2015). Na literatura, muitas abordagens demonstram uma preocupação com a redução do lead time, dentre elas destacam-se o Quick Response Manufacturing (QRM) (SAES; GODINHO FILHO, 2011). 
Quadro 1 - Níveis de Maturidade e suas características

\begin{tabular}{|c|c|}
\hline $\begin{array}{c}\text { Nível de } \\
\text { Maturidade }\end{array}$ & Características \\
\hline Inicial & $\begin{array}{l}\text { Práticas de gestão não estabelecidas; Postura reativa aos compromissos; } \\
\text { Planejamento ineficiente; Ambiente instável para desenvolvimento de } \\
\text { processos. }\end{array}$ \\
\hline Gerenciado & $\begin{array}{l}\text { Processos e projetos planejados, executados, medidos e controlados; } \\
\text { Compromissos estabelecidos conforme as necessidades dos stakeholders; } \\
\text { Requerimentos, produtos e serviços gerenciados; Validação do atendimento } \\
\text { aos requisitos, padrões e objetivos por revisão dos stakeholders; Práticas } \\
\text { existentes são mantidas; Status dos produtos e serviços são visíveis para a } \\
\text { gerência em pontos específicos }\end{array}$ \\
\hline Definido & $\begin{array}{l}\text { Processos padrão caracterizados, documentados, compreendidos, } \\
\text { integrados, descritos conforme padrões, procedimentos, ferramentas, } \\
\text { métodos; Gerência estabelece objetivos de processo baseados no conjunto } \\
\text { de processos padrão e assegura-se que eles são seguidos de forma } \\
\text { apropriada; Programa de treinamento para garantir conhecimentos e } \\
\text { habilidades }\end{array}$ \\
\hline $\begin{array}{l}\text { Quantitativamente } \\
\text { Gerenciado }\end{array}$ & $\begin{array}{l}\text { Objetivos quantitativos de qualidade para os produtos e desempenho de } \\
\text { processos são baseados nas necessidades dos clientes, usuários finais e } \\
\text { da própria organização; Produtividade e qualidade medidas em atividades } \\
\text { importantes em todos os projetos, como processo gerencial; Produtos e } \\
\text { processos compreendidos em termos estatísticos, reduzindo a variação no } \\
\text { desempenho, e gerenciados ao longo de toda a vida; Riscos na introdução } \\
\text { de um novo produto conhecidos e gerenciados }\end{array}$ \\
\hline Em otimização & $\begin{array}{l}\text { Estabelecidos objetivos mensuráveis de melhoria de processos, } \\
\text { continuamente revisados para refletir mudanças nos objetivos de negócio; A } \\
\text { organização inteira está focada na melhoria contínua do desempenho de } \\
\text { processo, por melhoria incremental e por inovações tecnológicas; Dados } \\
\text { sobre a efetividade dos processos usados para realizar análises de custo- } \\
\text { benefício das novas tecnologias e das mudanças propostas; Lições } \\
\text { aprendidas são disseminadas para outros projetos; Há otimização de } \\
\text { processos, com a participação e empowerment dos trabalhadores, alinhada } \\
\text { com os objetivos e valores da organização e seus negócios. }\end{array}$ \\
\hline
\end{tabular}

Fonte: (QUINTELLA; ROCHA, 2007)

\subsection{Quick Response Manufacturing (QRM)}

Em 1998, Rajan Suri apresentou o Quick Response Manufacturing (QRM) como uma aplicação específica para a redução de lead time em todas as operações de uma empresa (SURI, 1998; FERNANDES; CARMO-SILVA, 2006). Desde o seu surgimento, o QRM já foi implementado com sucesso em mais de 200 empresas nos Estados Unidos e Europa (SAES; GODINHO FILHO, 2011; LIMA et al. 2013) e por isso, começou a ganhar espaço na literatura nacional e internacional (GODINHO FILHO; SAES, 2013; LIMA et al. 2013; MELLO et al. 2016; GOMÉZ; GODINHO FILHO, 2016; GODINHO FILHO et al. 2016). 
Essa abordagem é bem estruturada e eficaz para a redução de lead time em sistemas que operam em uma base Make-to-Order (MTO), de baixo volume e alta variedade de produtos ou mesmo para empresas que trabalham em Engineer-toOrder (ETO) (FERNANDES; CARMO-SILVA, 2006; MACIEL NETO; GODINHO FILHO, 2011). Ela é fundamentada nos conceitos da Teoria de Filas e da Dinâmica de Sistemas para formular um conjunto de princípios para a reestruturação do chão de fábrica e práticas gerenciais. Além disso, a QRM apresenta uma nova abordagem que planeja e controla o fluxo de materiais em ambientes de manufatura com um grande mix de produtos, além de propor novos indicadores de desempenho (GODINHO FILHO et al. 2016).

O QRM é implementado por meio de um projeto que segue uma metodologia de quatro fases: definição do problema, coleta e análise de dados, proposição de melhorias e análise dos resultados esperados (LIMA et al. 2013). Na etapa de definição do problema e coleta e análise de dados, o QRM propõe o uso da ferramenta Manufacturing Critical-Path Time (MCT), que é a quantidade de tempo, medida a partir da criação de um pedido, passando pelo caminho crítico, até que a primeira peça desta ordem seja entregue ao cliente (ERICKSEN et al., 2007). Além disso, o MCT fornece também uma estimativa do lead time do processo, dividindo as atividades em produtivas que agregam valor (touch time) e as que não agregam valor (tempo em branco) (SURI, 2010). A terceira etapa, proposição de melhorias é embasada em 10 princípios-chave (Quadro 1) (SAES; GODINHO FILHO, 2011). E por fim, na fase de análise dos resultados esperados pode-se utilizar uma série de métodos empregados em trabalhos de Engenharia de Produção.

O QRM concentra-se em reduzir os prazos de entrega em todas as atividades de produção (FERNANDES; CARMO-SILVA, 2006), podendo ser aplicado em quatro áreas: manufatura, cadeia de suprimentos, introdução de novos produtos e em operações de escritório (SAES; GODINHO FILHO, 2011). 
Quadro 2 - Princípios-chave do QRM

\begin{tabular}{|c|c|c|}
\hline Número & Princípios & Definição \\
\hline 1 & $\begin{array}{l}\text { Encontre maneiras totalmente } \\
\text { novas para executar os trabalhos, } \\
\text { com foco na redução de lead time; }\end{array}$ & $\begin{array}{l}\text { Retirar o tempo do sistema requer repensar } \\
\text { completamente como a produção, } \\
\text { fornecimento de materiais e a gestão são } \\
\text { organizados }\end{array}$ \\
\hline 2 & $\begin{array}{l}\text { Planejar a capacidade dos recursos } \\
\text { críticos em } 80 \% \text { ou até mesmo } \\
70 \% \text {; }\end{array}$ & $\begin{array}{l}\text { O QRM recomenda que se procure obter } \\
\text { ganhos de flexibilidade e agilidade em vez de } \\
\text { maximizar o uso de equipamentos e de } \\
\text { funcionários }\end{array}$ \\
\hline 3 & $\begin{array}{l}\text { Medir a redução de lead time e } \\
\text { fazer desta a principal medida de } \\
\text { desempenho; }\end{array}$ & $\begin{array}{l}\text { A redução do lead time deve ser a principal } \\
\text { medida de desempenho }\end{array}$ \\
\hline 4 & $\begin{array}{l}\text { Medir e recompensar as reduções } \\
\text { de lead time e não entregas; }\end{array}$ & $\begin{array}{l}\text { A melhoria dos indicadores tradicionais será } \\
\text { consequência da estratégia focada no tempo }\end{array}$ \\
\hline 5 & $\begin{array}{l}\text { Use o MRP apenas nos níveis mais } \\
\text { altos de planejamento de produção } \\
\text { e materiais }\end{array}$ & $\begin{array}{l}\text { O QRM recomenda o uso do POLCA, que } \\
\text { combina recursos de MRP e Kanban }\end{array}$ \\
\hline 6 & $\begin{array}{l}\text { Motivar os fornecedores a } \\
\text { implementar o QRM }\end{array}$ & $\begin{array}{l}\text { É essencial que a empresa trabalhe com } \\
\text { fornecedores conscientes da importância do } \\
\text { tempo e buscando reduzir o lead time em suas } \\
\text { operações }\end{array}$ \\
\hline 7 & $\begin{array}{l}\text { Educar os clientes sobre } 0 \\
\text { programa QRM e negociar um } \\
\text { cronograma de redução do } \\
\text { tamanho dos lotes a preços } \\
\text { razoáveis; }\end{array}$ & $\begin{array}{l}\text { E necessário demonstrar ao cliente como o } \\
\text { QRM Ihes permitirá receber lotes pequenos, } \\
\text { rapidamente sem aumentar os preços }\end{array}$ \\
\hline 8 & $\begin{array}{l}\text { Eliminar as fronteiras funcionais por } \\
\text { meio da criação de células de } \\
\text { escritório de resposta rápida }\end{array}$ & $\begin{array}{l}\text { O QRM recomenda o uso de células também } \\
\text { nas operações do escritório, não apenas no } \\
\text { chão de fábrica }\end{array}$ \\
\hline 9 & $\begin{array}{l}\text { A razão para a adoção de um } \\
\text { projeto QRM é a redução do tempo } \\
\text { para a criação de sucesso de longo } \\
\text { prazo }\end{array}$ & $\begin{array}{l}\text { A verdadeira razão para a adoção do QRM é } \\
\text { tornar a empresa competitiva no longo prazo } \\
\text { através da competição baseada no tempo }\end{array}$ \\
\hline 10 & $\begin{array}{l}\text { O maior desafio do programa QRM } \\
\text { não envolve a tecnologia, mas a } \\
\text { mudança de mentalidade } \\
\text { organizacional. }\end{array}$ & $\begin{array}{l}\text { A mudança de uma mentalidade baseada na } \\
\text { eficiência e no custo para uma mentalidade } \\
\text { baseada na redução do lead time só ocorre } \\
\text { através do treinamento e conscientização }\end{array}$ \\
\hline
\end{tabular}

Fonte: Godinho Filho et al. (2016).

\subsubsection{QRM em operações de escritório}

As operações de escritório envolvem todas as atividades desenvolvidas pela empresa para o processamento de um pedido, que não ocorrem no chão de fábrica (LIMA et al. 2013). As atividades de escritório que mais impactam no lead time são: desenvolvimento de cotação, processamento de pedidos, novos projetos e desenvolvimento de produtos. Embora negligenciados como fonte de melhorias, as operações de escritório podem consumir mais da metade do lead time nas 
empresas, ser responsável por mais de $25 \%$ dos custos, influenciar a taxa de captação de pedidos e impactar no market share da empresa (SURI, 2010).

Suri $(1998,2010)$ explica como o lead time vai aumentando ao longo do tempo nas operações de escritório, o que ele chama de "Espiral do Tempo de Resposta" (Figura 1). A espiral começa quando os departamentos são requisitados a definir a data de determinada atividade (lead time inicial). Com base em problemas de projetos passados, os departamentos inserem tempos de segurança, o que gera perda de foco e consequentemente, acúmulo de trabalhos. O problema é agravado pelos erros de planejamento, ou quando para garantir o pedido, o departamento de vendas combina com o cliente a entrega do produto em tempo mais curto, o que faz com que trabalhos sejam acelerados (hot jobs), levando em atrasos nos trabalhos regulares. Baseado nessas ocorrências, que resultam em entregas atrasadas, o Departamento de Planejamento decide adicionar mais tempo de segurança ao lead time. Assim, a espiral recomeça e o lead time aumenta cada vez mais.

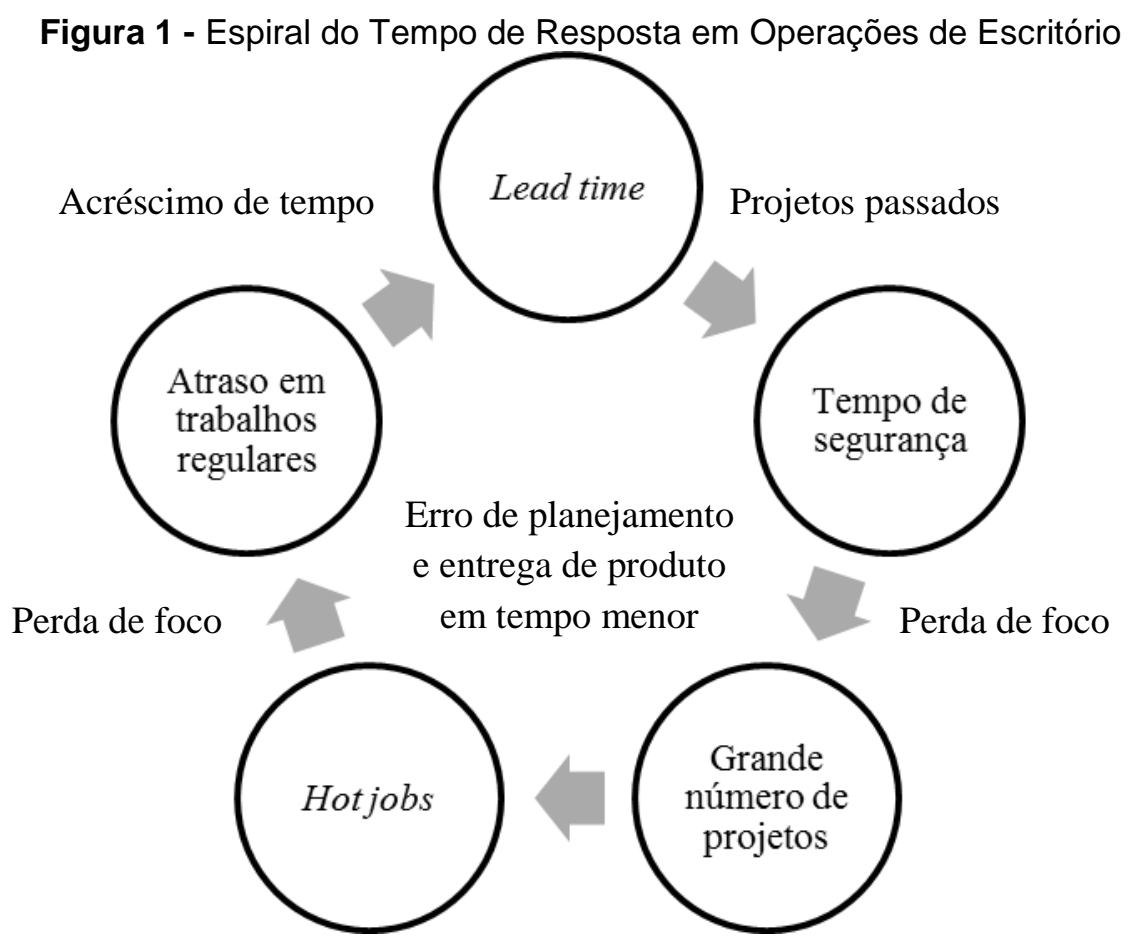

Fonte: Adaptado de Suri $(1998,2010)$

As raízes da Espiral do Tempo de Resposta estão na mentalidade das empresas baseadas na escala e custo, que divide as tarefas de escritório em 
pequenas etapas para a contratação de mão de obra barata. O problema é que essa estrutura cria muitos departamentos funcionais e cada tarefa deve passar por muitas pessoas para serem processadas. Além disso, a ênfase em redução de custos faz os departamentos funcionarem com alta utilização, gerando acúmulo de trabalho, problemas de qualidade e maiores custos para toda a empresa (SURI, 2010).

Para eliminar a Espiral do Tempo de Resposta, a mentalidade baseada no custo deve ser substituída pela mentalidade baseada no tempo (SURI, 1998). Uma forma de se alcançar esse objetivo é através do primeiro princípio do QRM: "Encontrar maneiras totalmente novas para completar uma tarefa, com foco principal na minimização do MCT" (SURI, 2010). Dentre as práticas que podem influenciar nessa redução estão: combinação e eliminação de passos, redesenho, fluxo contínuo de trabalho, novas tecnologias de informação e ferramentas, superar os pensamentos tradicionais (alta utilização, passos de aprovação, planejamento e controle complexos, etc).

Suri (2010) ainda atenta para a necessidade de se determinar um Focused Target Market Segment (FTMS) para se iniciar um projeto QRM. Para isso, inicialmente deve-se identificar um segmento de mercado no qual há uma oportunidade de aplicação da estratégia QRM, ou seja, buscar um cliente que seja sensível à redução de lead time. Identificado esse segmento, deve-se definir o foco do projeto, ou seja, o produto ou o processo cuja redução de lead time trará mais benefícios para a empresa.

A redução de lead time do PDP oferece a empresa ganhos de mercado e aumento de lucratividade. Assim, SURI (2010) propõe oito princípios gerenciais para acelerar o desenvolvimento de produtos: (1) criar senso de urgência no projeto através da conscientização da empresa; (2) implantar a gestão de projetos e métodos de caminhos críticos; (3) realizar controle de escopo do projeto, evitando a inserção de mudanças eventuais; (4) facilitar a troca de informações e interações mais frequentes; (5) criar uma infraestrutura para apoiar a troca de informações; (6) realizar parcerias com fornecedores e consumidores, para entender suas restrições e necessidades; (7) usar de organizações virtuais, aliança com concorrentes e cooperação para garantir aumento de competitividade; e (8) aprender com cada projeto, criando uma gestão do conhecimento. 


\section{MÉTODO}

O presente estudo utilizou como procedimentos metodológicos, segundo Berto e Nakano (1998), a pesquisa teórico-conceitual e o estudo de caso. A pesquisa seguiu a metodologia específica proposta para a implementação de um projeto QRM, sendo composta por quatro etapas (Figura 2). A primeira etapa consiste na definição do problema para análise da situação atual da empresa. Para isso foram elaborados o fluxograma do processo de desenvolvimento de novos produtos e o mapeamento do $\mathrm{MCT}$, a partir de entrevistas com o dois funcionários do departamento de desenvolvimento de produtos e planilhas de tempos e movimentos desenvolvida pela equipe de recursos humanos da empresa. $\mathrm{Na}$ segunda etapa, coleta e análise dos dados, foi utilizada a técnica de brainstorming para geração de novas idéias a partir de discussão em grupo composto por gerentes dos departamentos de desenvolvimento de produto, de produção, de projetos, marketing, laboratório, qualidade e assuntos regulatórios, totalizando nove funcionários. Esses funcionários trabalham na empresa a mais de cinco anos e estão a pelo menos dois envolvidos no desenvolvimento de novos produtos. $O$ brainstorming foi conduzido por meio de um roteiro de entrevista elaborado para identificar as causas do lead time. A terceira etapa consiste na proposição de melhorias, apresentando soluções potenciais para a resolução de problemas apresentados, usando-se principalmente do know-how dos funcionários do departamento de desenvolvimento de projeto. Tanto, as reuniões, as entrevistas foram gravadas, transcritas e analisadas por meio da abordagem de conteúdo. Por fim, a análise dos resultados esperados (quarta etapa), identifica a possibilidade de implementação das propostas de melhorias por meio da análise custo/benefício, sendo que essa técnica foi utilizada para verificar se a aplicação do QRM seria viável economicamente para empresa. 
Figura 2 - Procedimentos metodológicos do estudo.

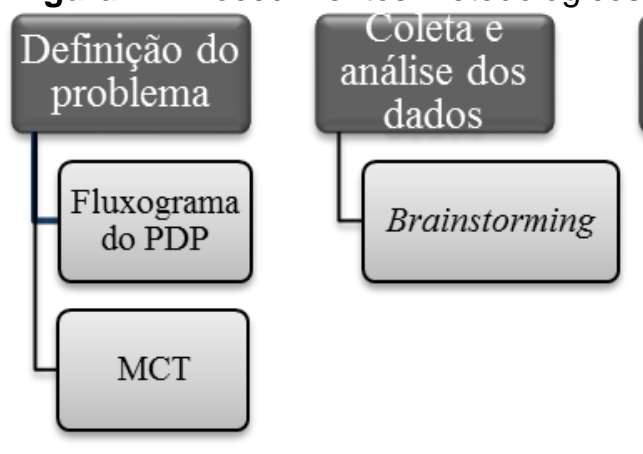

Proposição
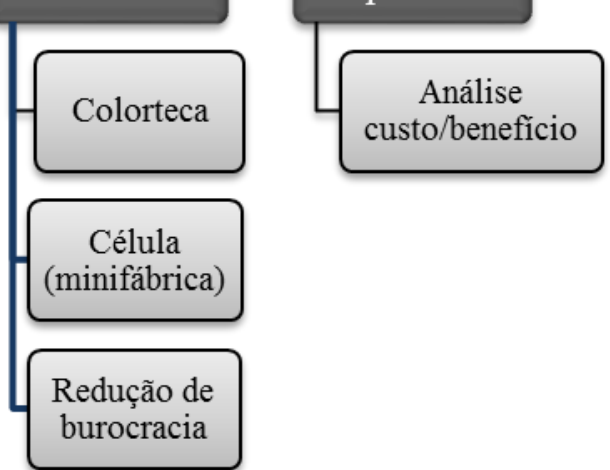

Fonte: Adaptado de Lima et al. (2013)

\section{RESULTADOS E DISCUSSÕES}

Nessa seção serão apresentados os resultados obtidos no estudo. A primeira subseção apresenta a empresa onde o estudo foi aplicado, ressaltando principalmente a composição do lápis madeira e o fluxograma do processo. A segunda subseção trata da aplicação do QRM na empresa estudada, e é composta por quatro subdivisões: definição do problema, coleta e análise de dados, propostas e melhorias e análise dos resultados esperados.

\subsection{A empresa estudada}

A empresa estudada foi fundada em 1761 na Alemanha, possui 14 fábricas no mundo, onde emprega mais de 7.000 colaboradores. Sua principal subsidiária está localizada no Brasil, formada por quatro unidades, uma de produção de mudas e operações florestais com industrialização da madeira em Prata (MG), uma de preservação permanente em Morretes (PR), uma de fabricação de produtos plásticos em Manaus (AM) e uma de produção em São Carlos (SP).

O estudo de caso foi conduzido na unidade de produção localizada em São Carlos. Ela emprega atualmente mais de 2.700 funcionários e produz mais de mil itens que abastecem o mercado interno e são exportados para mais de 70 países. A produção da empresa concentra-se em materiais de escrita (lápis, canetas, canetinhas, marcadores de texto, borracha, apontador, corretivos, lapiseiras, giz de 
cera, colas, tintas), no entanto, os lápis pretos e coloridos são os principais negócios, tendo capacidade de produção mundial de mais de dois bilhões por ano.

No departamento de desenvolvimento de produto trabalham cinco funcionários, que tem como principais atividades, recebimento, análise, definição, coordenação e acompanhamento de todas as etapas do desenvolvimento de um novo produto. Foi definido como FTMS, o lápis madeira para olhos, que representa $70 \%$ do faturamento da empresa.

Os clientes da empresa são multinacionais e organizações nacionais de médio e grande porte, que estão no mercado entre 10 e 130 anos. O número de funcionários e o faturamento dessas empresas são muito distintos, podendo variar de 122 até 50 mil funcionários e de $R \$ 200$ milhões a $R \$ 9$ bilhões anuais. $A$ linha de lápis de olhos compreendendo linhas com diferentes cores, tonalidades, textura, design, diâmetro, tamanho, acabamento, embalagem, duração da aplicação e funções.

O lápis é formado pela mina (1), que é a parte que entrará em contato com os olhos. Ela é envolvida por uma madeira que recebe uma camada de verniz em sua superfície (2) e em uma de suas extremidades (gota) (3). Sobre o verniz são inseridas marcações legais (4), uma tampa plástica (5) para evitar a contaminação do produto e uma embalagem (6) (Figura 2).

Figura 2 - Composição do lápis de olho

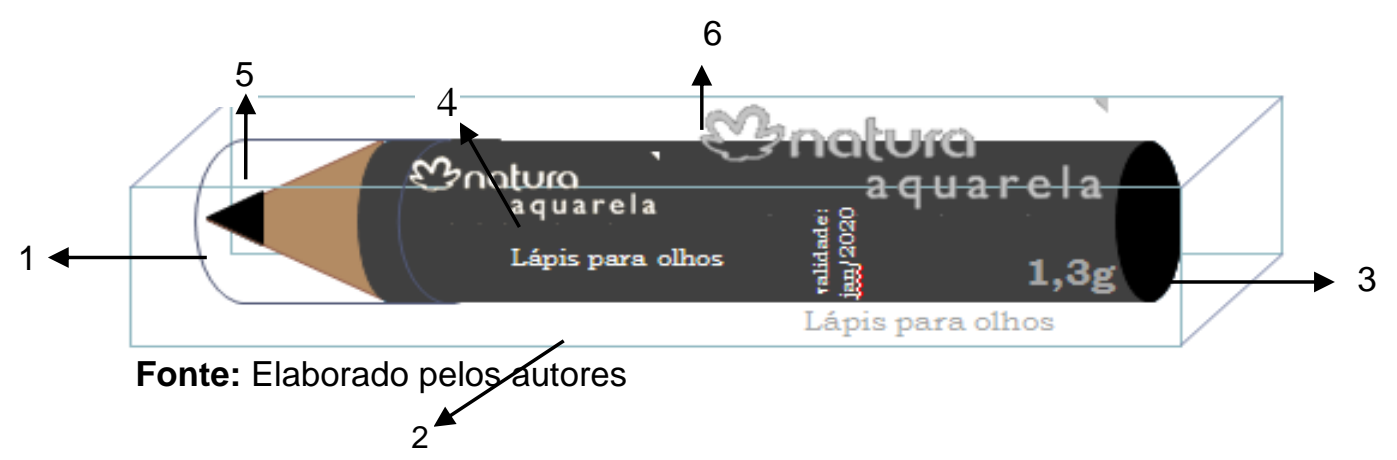

\subsection{Definição do problema}

Nessa seção, há a exposição das ferramentas utilizadas na etapa de definição do problema. Para tanto, foram utilizadas as ferramentas fluxograma do PDP e MCT.

Revista Produção Online. Florianópolis, SC, v. 18, n. 2, p. 504-531, 2018. 


\subsubsection{Fluxograma do PDP}

O processo inicia-se com o contato do vendedor com o cliente, o qual elabora um documento com as especificações do produto. Uma pré-análise desse documento é realizada pela empresa para verificar a disponibilidade e a capacidade da produção. Depois de confirmado, inicia-se o desenvolvimento de amostras de cores da mina e em paralelo, o texto legal (exigência legal do país onde o produto será vendido), a arte (que será inserida no produto e na sua embalagem), o verniz e a gota e a matéria-prima (madeira) é analisada. Feito isso, são desenvolvidos em sequencia a embalagem, a arte secundária, os testes da gravação e o plástico (tampa) (que é realizado por uma empresa terceirizada). Todas as etapas mencionadas devem ter a aceitação do cliente. Depois disso, a qualidade da gravação é analisada, o Documento de Especificação do Produto (DEP) é elaborado e verificado e o produto é cadastrado no SAP da empresa. Por fim, o produto é inserido no sistema para, então, ser feita a programação da produção (Figura 3).

\subsubsection{MCT}

A Figura 4 apresenta o MCT do processo de desenvolvimento de um novo lápis de olho. A descrição de cada atividade com seu número correspondente foi exposta no Quadro 1. Vale à pena ressaltar que a barra azul da Figura 4 representa a quantidade de tempo da etapa em que há agregação de valor (touch time) e a vermelha o tempo em que não há agregação de valor (tempo em branco). 


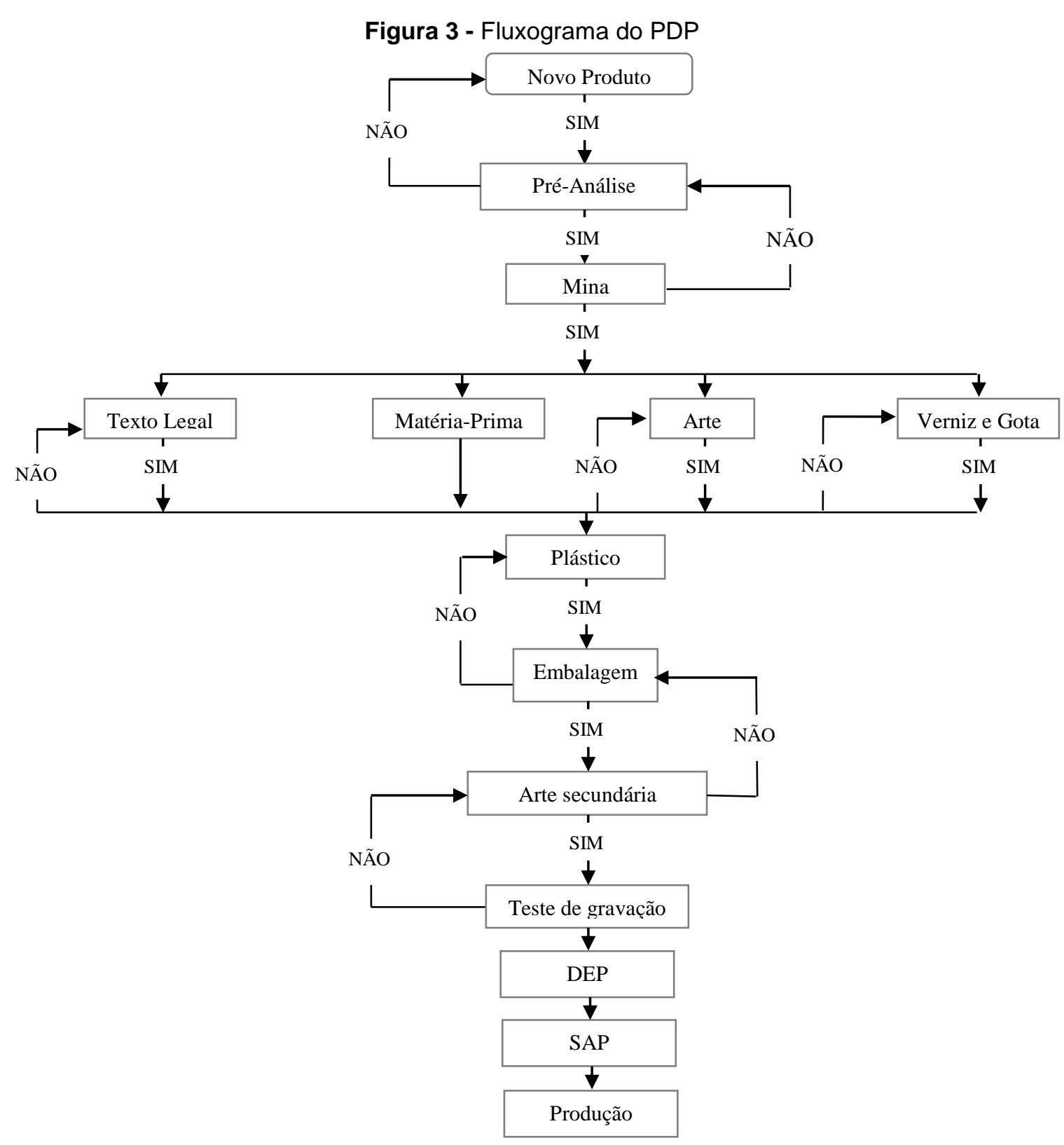

Fonte: Elaborado pelos autores, a partir dos dados da pesquisa

Revista Produção Online. Florianópolis, SC, v. 18, n. 2, p. 504-531, 2018. 


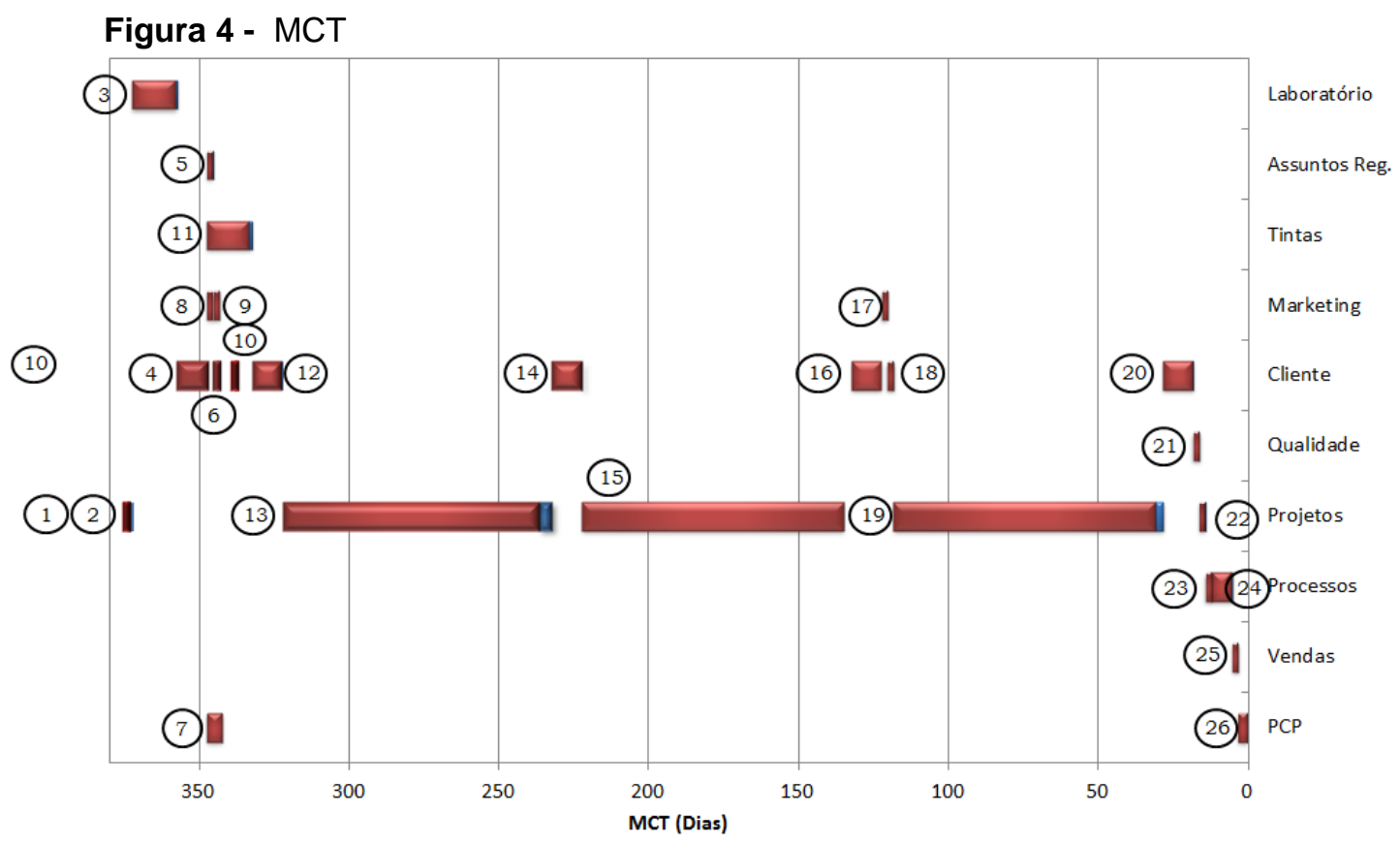

Fonte: Elaborado pelos autores, a partir dos dados da pesquisa

Portanto, a partir do MCT, obtiveram-se informações de como as atividades são realizadas e estabeleceu-se uma estimativa inicial de como se comporta o lead time entre as etapas do processo. Verificou-se que o tempo total gasto para a realização do processo de desenvolvimento de um novo lápis de olho é de 375 dias, sendo que $95,4 \%$ desse tempo não agrega valor ao produto. 
Quadro 1 - Descrição das etapas do processo de desenvolvimento do lápis de olho.

\begin{tabular}{|c|l|c|l|}
\hline $\mathbf{N} N$ & \multicolumn{1}{|c|}{ Atividade } & $\mathbf{N}$ & \multicolumn{1}{|c|}{ Atividade } \\
\hline $\mathbf{1}$ & Análise do Briefing & $\mathbf{1 4}$ & Aprovação do cliente \\
\hline $\mathbf{2}$ & Elaboração do Cronograma & $\mathbf{1 5}$ & Desenvolvimento da Embalagem \\
\hline $\mathbf{3}$ & Desenvolvimento da Mina & $\mathbf{1 6}$ & Aprovação do cliente \\
\hline $\mathbf{4}$ & Aprovação do cliente & $\mathbf{1 7}$ & Fluxo de arte secundaria \\
\hline $\mathbf{5}$ & Texto Legal & $\mathbf{1 8}$ & Aprovação do cliente \\
\hline $\mathbf{6}$ & Aprovação do cliente & $\mathbf{1 9}$ & Testes de Gravação \\
\hline $\mathbf{7}$ & Análise da Matéria-Prima & $\mathbf{2 0}$ & Aprovação do cliente \\
\hline $\mathbf{8}$ & Desenvolvimento da Arte & $\mathbf{2 1}$ & Análise de qualidade da gravação \\
\hline $\mathbf{9}$ & Fluxo de arte primaria & $\mathbf{2 2}$ & Elaboração do DEP \\
\hline $\mathbf{1 0}$ & Aprovação do cliente & $\mathbf{2 3}$ & Verificação do DEP \\
\hline $\mathbf{1 1}$ & Desenvolvimento do Verniz e Gota & $\mathbf{2 4}$ & Cadastro SAP e Fluxo do cadastro \\
\hline $\mathbf{1 2}$ & Aprovação do cliente & $\mathbf{2 5}$ & Pedido no sistema \\
\hline $\mathbf{1 3}$ & Desenvolvimento do Plástico & $\mathbf{2 6}$ & Programação da produção pelo PCP \\
\hline
\end{tabular}

Fonte: Elaborado pelos autores, a partir dos dados da pesquisa

\subsection{Coleta e análise dos dados}

A partir do MCT, foi possível identificar cinco áreas de melhoria: desenvolvimento da mina (3), do verniz e da gota (11), do plástico (13), da embalagem (15) e teste de gravação (19). Essas áreas foram selecionadas por serem responsáveis por mais de $80 \%$ do lead time (300 dias) e por elas apresentarem mais de $90 \%$ de tempo em branco.

O lead time das atividades de desenvolvimento da mina, de verniz e gota acontece por motivos semelhantes: grande número de submissões, pouco tempo para $\mathrm{P} \& \mathrm{D}$, especialistas pouco focados, grande tempo de espera devido à carga de trabalho e retrabalhos. No desenvolvimento do plástico, o lead time ocorre por causa dos tempos despendidos do envio das amostras do fornecedor para a empresa e da empresa para aprovação do cliente, além da burocracia do departamento de compras para a realização de orçamentos, notas fiscais e contato com o fornecedor. Já o lead time do desenvolvimento de embalagem ocorre devido ao tempo de transporte da matéria-prima (fornecedores internacionais) e pelo tempo de espera para a amostra ser incluída no planejamento da fábrica. Finalmente, o lead time dos testes de gravação deve-se, além da burocracia interna, ao tempo de espera para a 
amostra do produto ser incluída no planejamento da fábrica para realização dos testes e análise da qualidade da gravação.

\subsection{Proposta de melhorias}

Em reunião com o pessoal do departamento de projetos e também utilizando o primeiro princípio QRM de Suri (1998) - "encontrar maneiras totalmente novas para realizar uma tarefa, com foco principal na redução de lead time" conseguiu-se determinar três propostas de melhorias: colorteca, desenvolvimento de uma célula (minifábrica) para testes e redução da burocracia.

\subsubsection{Colorteca}

A Colorteca consiste em um arquivo físico com amostras de cores já desenvolvidas pela empresa (FREITAS et al.2016). Por meio dela, o vendedor, em seu contato inicial com o cliente apresenta as opções de cores existentes de mina, verniz, gota, e plástico, através de um tablet, podendo gerar o pedido na primeira visita. Assim, pode-se eliminar a necessidade de novos desenvolvimentos, ou, pelo menos, se consegue especificar melhor a variação da cor desejada pelo cliente, reduzindo o tempo despendido com esse processo.

\subsubsection{Célula (Minifábrica)}

As células do ambiente de produção consistem em conjunto de máquinas, arranjadas de acordo com o fluxo do produto para minimizar o tempo do trabalhador. A célula é operacionalizada por uma equipe multifuncional que é treinada para desempenhar diversas atividades, sendo ela dedicada a uma família de produtos que precisam de operações similares e buscam completar todas as atividades dentro da célula (SURI, 1998).

Utilizando-se dessa ferramenta, foi proposta a implantação da minifábrica, entretanto, somente para realizar os testes de gravação no lápis e da embalagem. Atualmente, esses testes são feitos nas mesmas máquinas da produção da fábrica 
e, para realizá-los, é necessário que o PCP altere seu planejamento e incluam os testes na programação da fábrica. Com isso, a cada novo teste, a produção normal da fábrica é interrompida e são gastos tempos com setup das máquinas. A proposta de implantação da minifábrica envolve o aproveitamento das máquinas antigas da empresa e que não estão sendo utilizadas e dispostas em proximidade. Com isso, não seria mais necessário interromper a produção para a realização desses testes e se teria maior agilidade no processo de desenvolvimento, além de garantir maior qualidade aos produtos, uma vez que os funcionários do departamento de desenvolvimento de novos produtos teriam maior controle sobre o processo e poderiam corrigir os problemas em um tempo menor.

\subsubsection{Redução da burocracia interna}

A burocracia pode ser entendida como um tipo de poder capaz de racionalizar os processos organizacionais utilizados nas relações sociais (SARAIVA, 2002). Segundo Paiva (2009), o aumento dos níveis de burocracia tem a ver com o crescimento de organizações de estruturas hierarquizadas, onde rotinas racionais e impessoais prevalecem. Dessa forma, a burocracia tem como intuito controlar legalmente os processos por meio da emissão de documentos emitidos por órgãos, secretarias ou departamentos específicos. Apesar disso, ela também tende a ser demorada e onerosa.

Outra proposta de melhoria está relacionada à redução da burocracia interna, que está presente em praticamente todo o processo de desenvolvimento de novos produtos. As documentações e solicitações que precisam do aval dos departamentos, as incessantes aprovações do cliente para o desenvolvimento de todos os componentes do lápis e, as aprovações da empresa em relação à matériaprima enviada pelo fornecedor são as grandes responsáveis por parte do lead time. Apesar disso, essas tarefas podem ser repensadas e um novo fluxo de atividades pode ser implantado, reduzindo o tempo de execução do processo. Para isso, devese dar maior autonomia aos funcionários (empowerment) e exigir a certificação da qualidade dos fornecedores. 
O empowerment é uma abordagem de projeto de trabalho que objetiva a delegação de poder de decisão, autonomia e participação dos funcionários na administração das empresas (RODRIGUES; SANTOS, 2001). Dessa forma, um funcionário pode modificar o seu trabalho e na forma como ele é desempenhado. $\mathrm{Na}$ empresa seria necessário o treinamento de funcionários do departamento de desenvolvimento de produto para elaboração de documentos e realização de atividades que atualmente são desenvolvidas em outros departamentos, o que garantiria maior agilidade nas tomadas de decisão do PDP.

A certificação pode ser definida como um atestado, emitido por órgãos específicos, de que $O$ atendimento aos requisitos desejados foi demonstrado, informando assim, a credibilidade de uma empresa (PEREIRA SOUZA et al. 2012). A ISO 9001 é um padrão certificável de qualidade que foca na eficácia dos processos a partir de melhores especificações de procedimentos, treinamento da mão de obra e da melhoria contínua (MAEKAWA et al. 2013). Dessa forma, a certificação ISO 9001 do fornecedor é importante para demonstrar a qualificação de seus processos e produtos, garantindo assim, que ele seja apto a comunicar diretamente com os clientes para aprovação de amostras, sem a necessidade de intermediação da empresa.

\subsubsection{Análise dos resultados esperados}

Para analisar os resultados esperados com a implantação das propostas de melhoria, foram levantados os custos de implementação e os benefícios de cada proposta, seguido dos benefícios gerais com a implantação do projeto QRM (Tabela 1). 
Tabela 1 - Custo de implementação das três propostas de melhoria.

\begin{tabular}{cccc}
\hline PROPOSTA & QUANTIDADE & $\begin{array}{c}\text { PREÇO } \\
\text { UNITÁTIO (R\$) }\end{array}$ & TOTAL (R\$) \\
\hline Colorteca & 10.000 & 1 & 10.000 \\
Pantones & 8 & 600 & 4.800 \\
Tablets & 4 & 10.000 & 40.000 \\
Armário & 1 & 12.000 & 12.000 \\
Estagiário (1 ano) & & & 120.000 \\
Minifábrica & 3 & 40.000 & 40.000 \\
Manutenção de máquinas & 1 & 40.000 & 40.000 \\
Espaço físico & 1 & 40.000 & \\
Aquisição de materiais e & & & 20.000 \\
ferramentas & 10 & 2.000 & $\mathbf{R} \mathbf{2 8 6 . 8 0 0 , 0 0}$ \\
Redução da burocracia & - & - & \\
Treinamento dos funcionários & & & \\
VALOR TOTAL & & &
\end{tabular}

Fonte: Elaborado pelos autores, a partir dos dados da pesquisa

O custo de implantação da Colorteca é composto pela confecção dos pantones (amostras de cores) que serão apresentadas ao cliente; a aquisição de tablets para apresentação das opções de cores existentes na fábrica; a compra de armários para guardar a coleção; e pagamento de salário do estagiário de biblioteconomia, para catalogar todas as cores no mostruário. Em relação aos custos da minifábrica, a empresa precisa fazer manutenção nas máquinas e organizar o espaço físico onde ela será instalada e para a aquisição de materiais e ferramentas. Os custos para a redução da burocracia estão relacionados à realização de treinamentos dos funcionários da equipe de projetos para dar empowerment aos funcionários para tomar decisões, sem passar pelos demais departamentos da empresa; eliminação de atividades que não agregam valor e a comunicação direta entre o fornecedor e o cliente a respeito das amostras de plástico desenvolvidas, sem a necessidade de intermediação da empresa. Assim, o investimento total das três propostas é de $R \$ 286.800,00$ reais. 
Com essas propostas, existirão dois tipos de projetos: os que não precisarão de desenvolvimento e os que ainda precisarão de algum tipo de desenvolvimento. Estima-se uma redução de lead time de 70\% (264 dias) em relação ao lead time total do processo (375 dias) para os projetos que não precisarão de desenvolvimento, conforme Tabela 2, uma vez que os projetos não passarão pelas atividades de desenvolvimento de mina, de verniz e gota e plástico. As atividades de desenvolvimento da embalagem e testes de gravação terão seu tempo reduzido através da redução da burocracia e implantação da minifábrica.

Tabela 2- Produtos que não precisarão de desenvolvimento

\begin{tabular}{|c|c|c|c|c|c|}
\hline Atividades desenvolvidas & $\begin{array}{c}\text { Lead time } \\
\text { total } \\
\text { (dias) }\end{array}$ & $\begin{array}{c}\text { Touch } \\
\text { time } \\
\text { (dias) }\end{array}$ & $\begin{array}{c}\text { Tempo } \\
\text { em } \\
\text { branco } \\
\text { (dias) }\end{array}$ & $\begin{array}{l}\text { Lead time } \\
\text { esperado }\end{array}$ & $\begin{array}{l}\text { Redução de } \\
\text { lead time } \\
(\%)\end{array}$ \\
\hline Mina & 15 & 0,9 & 14,1 & 0 & 100 \\
\hline Verniz e gota & 15 & 1,2 & 13,8 & 0 & 100 \\
\hline Plástico & 90 & 4,5 & 85,5 & 0 & 100 \\
\hline Embalagem & 90 & 4,5 & 85,5 & 27 & 70 \\
\hline Teste de gravação & 90 & 0,9 & 89,1 & 9 & 90 \\
\hline Total & 300 & 12 & 288 & 36 & $70 \%$ \\
\hline
\end{tabular}

Fonte: Elaborado pelos autores, a partir dos dados da pesquisa.

A Tabela 3 mostra a redução de lead time para os projetos que ainda precisarão de algum tipo de desenvolvimento. Com a implantação da Colorteca, os vendedores conseguirão detalhar melhor as expectativas dos clientes em relação às cores desejadas e os laboratórios terão mais tempo para novos desenvolvimentos. A implantação da minifábrica possibilitará a redução do lead time das atividades de desenvolvimento da embalagem e testes de gravação. Finalmente, a redução da burocracia contribuirá para a redução de lead time de todas as atividades críticas. Estima-se que a redução de lead time para os projetos que ainda precisarão de desenvolvimento seja de $45 \%$ (168 dias). 
Tabela 3 - Produtos que ainda precisarão de desenvolvimento

\begin{tabular}{|c|c|c|c|c|c|}
\hline Atividades desenvolvidas & $\begin{array}{c}\text { Lead time } \\
\text { total } \\
\text { (dias) }\end{array}$ & $\begin{array}{l}\text { Touch } \\
\text { time } \\
\text { (dias) }\end{array}$ & $\begin{array}{l}\text { Tempo em } \\
\text { branco } \\
\text { (dias) }\end{array}$ & $\begin{array}{c}\text { Lead } \\
\text { time } \\
\text { esperado }\end{array}$ & $\begin{array}{l}\text { Redução de } \\
\text { lead time } \\
\text { (\%) }\end{array}$ \\
\hline Mina & 15 & 0,9 & 14,1 & 7,5 & 50 \\
\hline Verniz e gota & 15 & 1,2 & 13,8 & 7,5 & 50 \\
\hline Plástico & 90 & 4,5 & 85,5 & 45 & 50 \\
\hline Embalagem & 90 & 4,5 & 85,5 & 45 & 50 \\
\hline Teste de gravação & 90 & 0,9 & 89,1 & 27 & 70 \\
\hline Total & 300 & 12 & 288 & 132 & $45 \%$ \\
\hline
\end{tabular}

Fonte: Elaborado pelos autores, a partir dos dados da pesquisa

Além disso, acredita-se que $40 \%$ dos projetos desenvolvidos pela empresa não precisarão mais de desenvolvimentos com a implantação da Colorteca. Para calcular a redução de lead time total deste projeto QRM, foi realizada uma média ponderada das reduções de lead time para os projetos que não precisarão de desenvolvimento ( $40 \%$ dos projetos; $70 \%$ de redução de lead time) e dos projetos que ainda precisarão de desenvolvimento (60\% dos projetos; $45 \%$ de redução de lead time). Com isso, espera-se que a implementação dessas propostas resulte em uma redução de $55 \%$ do lead time total do processo de desenvolvimento de novos lápis.

Segundo os dados fornecidos pela empresa, no ano de 2014, foram desenvolvidos 282 projetos. Considerando que um ano seja composto por 230 dias úteis, a empresa desenvolveu em média de 1,23 projetos por dia. Em 2015, somente no primeiro semestre, já foram completados 254 projetos, o que dá uma média de 2,21 projetos por dia, um crescimento de $80 \%$ na taxa de saída de projetos. Além disso, ao final do semestre, havia 26 projetos em fila. Com isso, percebe-se a necessidade da empresa se tornar mais ágil e conseguir uma taxa de saída (throughput) maior para atender a demanda crescente do mercado.

Utilizando-se a Lei de Litte, uma lei fundamental de dinâmica do sistema (system dynamics) que determina que o estoque em processo-work in process(WIP) é igual ao produto da taxa de saída do sistema (throughput) pelo lead time do sistema (Equação 1). Desta forma, com a redução proposta por esse projeto (55\%), 
o throughput da empresa aumentará quase $222 \%$, atendendo satisfatoriamente 0 aumento da demanda.

Lei de Little

$$
W I P=\text { throughput } \times \text { lead time }
$$

Apesar dos possíveis benefícios adquiridos com as propostas de melhorias, a sua implementação depende diretamente do retorno financeiro para a empresa. Desse modo, buscando demonstrar os custo/benefício das propostas, foram elaborados dois cenários econômicos, um pessimista e outro otimista. Sabendo-se que em 2014 a empresa desenvolveu 282 projetos e teve um faturamento de $R \$ 47$ milhões e que, no primeiro semestre de 2015 ela já foram desenvolvidos 254 projetos, gerou-se um cenário pessimista, considerando um aumento do número de projetos em 30\% em relação a 2014 (367 projetos), com um retorno financeiro 15\% maior ( $\mathrm{R}$ \$ 54,05 milhões); e um cenário otimista, com um crescimento do número de projetos de $80 \%$ em relação a 2014 (508 projetos), com um faturamento 40\% maior que o ano anterior ( $\mathrm{R} \$ 65,8$ milhões) (Gráfico 1).

Gráfico 1 - Quantidade de projetos e faturamento para o cenário pessimista e otimista

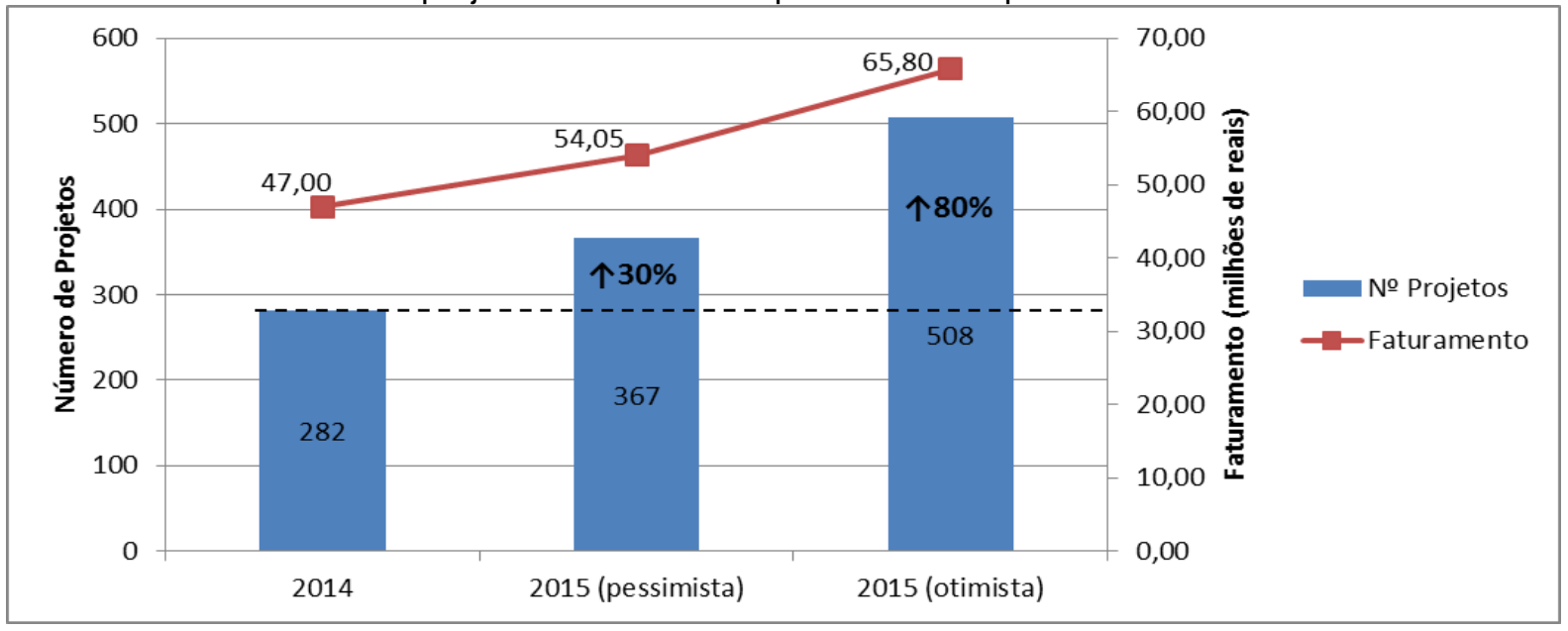

Fonte: Elaborado pelos autores, a partir dos dados da pesquisa.

Sendo assim, o investimento para a implantação das melhorias propostas $(R \$$ $268.800,00$ ) representa apenas 3,8\% do aumento do faturamento ( $\mathrm{R} \$ 7,05$ milhões) proporcionado pela redução de lead time no cenário pessimista, enquanto representa somente $1,4 \%$ do aumento do faturamento ( $\$$ \$18,8 milhões) no cenário 
otimista. Com isso, prova-se a viabilidade econômica da implantação do projeto QRM no processo de desenvolvimento de lápis para olhos.

\section{CONSIDERAÇÕES FINAIS}

Este trabalho teve como objetivo propor a aplicação da abordagem QRM para o processo de desenvolvimento de lápis para olhos em uma empresa fabricante de cosméticos. Seguindo essa abordagem foi possível identificar as principais atividades que contribuíam para o lead time do processo. A partir de reuniões com os funcionários da empresa foram elaboradas propostas de melhoria viáveis economicamente, com uma estimativa expressiva de $55 \%$ de redução de lead time.

Uma contribuição importante desta pesquisa foi à proposta de aplicação do QRM para o processo de desenvolvimento de novos produtos, área ainda carente na literatura. Além disto, este trabalho evidenciou a grande proporção de tempo que não agrega valor em operações de escritório, e mais especificamente, na área de desenvolvimento de produtos. Com este trabalho, também foi possível perceber o impacto do primeiro princípio QRM em atividades de escritório, sendo que a proposta de um mostruário de cores se mostrou muito eficaz para a redução de lead time, podendo eliminar totalmente três atividades que, juntas, representavam mais de $30 \%$ do MCT de todo o processo de desenvolvimento do lápis.

Uma limitação deste trabalho foi o acesso aos dados financeiros da empresa, para a análise mais precisa dos ganhos possibilitados pela implantação das propostas de melhoria. Apesar disso, com o treinamento dos gestores e funcionários para a busca constante pela redução de lead time e com a mudança das formas de incentivo, os benefícios dimensionados no projeto permitirão à empresa a alcançar sucesso a longo prazo.

Trabalhos futuros podem utilizar a pesquisa-ação para a análise da implantação do QRM e utilizar resultados reais como motivador para iniciar outros projetos na empresa estudada, inclusive no processo de produção do lápis para olhos. Além disso, o conteúdo pode ser utilizado em empresas que desejam melhorar seu tempo de resposta em operações de escritório e, principalmente para 
projeto e desenvolvimento de produtos, mostrando os passos básicos para implantação de um projeto QRM nessas áreas.

\section{REFERÊNCIAS}

ASSOCIAÇÃO BRASILEIRA DA INDÚSTRIA DE HIGIENE PESSOAL, PERFUMARIA E COSMÉTICOS (ABIHPEC). Panorama do setor em 2016. Disponível em:

https://abihpec.org.br/publicacao/panorama-do-setor-2016-2/ Acesso em: 5 abr. 2017.

ANDRADE, J. H. DE et al. Quick Response Manufacturing: Aplicação De Conceitos E Ferramentas Para a Redução Do Lead Time Na Manufatura De Bens. XXXI Encontro Nacional De Engenharia De Produção. Anais... Belo Horizonte: 2011. Disponível em: <http://www.abepro.org.br/biblioteca/enegep2011 TN STO 135856 17695.pdf>.

BERTO, R. M. V. DE S.; NAKANO, D. N. Metodologia da pesquisa e a engenharia de produção. XVIII Encontro Nacional de Engenharia de Produção. Anais... Niterói: 1998.

BERNARDI SOUZA, F.; MORAES, A. A. C. Análise da aplicação da gestão de projetos por corrente crítica no processo de desenvolvimento de produtos e na gestão de portfólio de um fabricante de aeronaves. Gestão \& Produção, v. 23, n.3, p. 473-485, 2016.

DOI: https://doi.org/10.1590/0104-530x1953-15

BOOTH, R. Agile manufacturing. Engineering Management Journal, v. 6, n. 2, p. 105-112, 1996. DOI: https://doi.org/10.1049/em:19960206

ERICKSEN, P.; STOFLET, N.; SURI, R. Manufacturing critical-path time (MCT): The QRM Metric for Lead Time. Technical Report, Certer for QRM, Wiscosin-Madison, 2007. Disponívelem: <http://www.Isoptimal.com/wordpress/wp-content/uploads/2012/08/mctdefinition-1.pdf>. Acesso em: 02 jul. 2015.

CAMPOS, S. U.; RIBEIRO, J. L. D. Um modelo de referência para o processo de desenvolvimento de produtos de empresas do setor moageiro de trigo. Produção, v. 21, n.3, p. 379-391, 2011. DOI: https://doi.org/10.1590/S0103-65132011005000036

ECHEVESTE, M. E.; ROZENFELD, H.; SONEGO, M. Potential application of Six Sigma tool in the integrated product development process. Journal of Brazilian Society of Mechanical Sciences and Engineering, v. 38, n.8, p. 2499-2511, 2016. DOI: https://doi.org/10.1007/s40430-016-0503-0

FERNANDES, N. O.; CARMO-SILVA, S. Generic POLCA-A production and materials flow control mechanism for quick response manufacturing. International Journal of Production Economics, v. 104, p. 74-84, 2006. DOI: https://doi.org/10.1016/j.ijpe.2005.07.003

FREITAS, D. C.; OLIVEIRA, L. G.; BERALDO, K. C.; GERIBELLO, C. P. CAETANO, M. B. P. Proposta de aplicação da abordagem Quick Response Manufacturing (QRM) no setor de desenvolvimento de produtos em uma empresa de cosméticos. In: XXXVI Encontro Nacional de Engenharia de Produção. Anais...João Pessoa, 2016. 
GODINHO FILHO, M.; MARCHESINI, A. G.; RIEZEBOS, J.; VANDAELE, N.; GANGA, G. M. $D$. The extent of knowledge of Quick Response Manufacturing principles: an exploratory transnational study. International Journal of Production Research, v.54, p. 1-21, 2016.

GODINHO FILHO, M.; GÓMEZ, F. J. Complementing lean with quick response manufacturing: case studies. International Journal of Advanced Manufacturing Technology, p.1-14, 2016.

GODINHO FILHO, M.; BARCO, C. F.; SAGAWA, J. K.; KINOSHITA, D.; PEIXOTO, M. G.M. Proposta De Aplicação Da Abordagem Quick Response Manufacturing Em Uma Empresa Do Setor Automobilístico. ENCONTRO NACIONAL DE ENGENHARIA DE PRODUÇÃO, 31. 2011. Anais... Belo Horizonte: 2011. Disponível em: <http://www.abepro.org.br/biblioteca/enegep2011 tn stp 135856 17792.pdf>.

GODINHO FILHO, M.; SAES, E. V. From time-based competition (TBC) to quick response manufacturing $(\mathrm{QRM})$ : The evolution of research aimed at lead time reduction. International Journal of Advanced Manufacturing Technology, v. 64, n. 5-8, p. 1177-1191, 2013. DOI: https://doi.org/10.1007/s00170-012-4064-9

IBGE - Instituto Brasileiro de Geografia e Estatística. Anuário Estatístico 11. Rio de Janeiro, IBGE, 2011. Disponível em:

<http://www.ibge.gov.br/home/estatistica/populacao/mapa mercado trabalho/default.shtm>. Acesso em: 29 jul. 2015.

JUGEND, D.; LUIS DA SILVA, S.; TOLEDO, J. C.; ONOYAMA, M. M. Evaluation of maturity levels in the management of product development: case studies in the capital goods industry. Product: Management \& Development, v. 8, n.2, p. 183-192, 2010.

LIZARELI, F. L.; TOLEDO, J. C. Práticas para a melhoria contínua do Processo de Desenvolvimento de Produtos: análise comparativa de múltiplos casos. Gestão \& Produção, v. 23, n.3, p. 1-21, 2016. DOI: https://doi.org/10.1590/0104-530x2240-15

LIMA, A. D.; BACHEGA, S. J.; GODINHO FILHO, M.; CRUZ, V. J. S.; ROSSI, J. M. Proposta de aplicação da abordagem Quick Response Manufacturing (QRM) para a redução do lead time em operações de escritório. Revista Produção, v. 23, n.1, p.1-19, 2013.

MACIEL NETO, J. D.; GODINHO FILHO, M. Integração entre o QRM e as melhores práticas em Gestão de Projetos. ENCONTRO NACIONAL DE ENGENHARIA DE PRODUÇÃO, 31., 2011. Anais... Belo Horizonte: 2011. Disponível em:

<http://www.abepro.org.br/biblioteca/enegep2011 TN STO 142899 18530.pdf>.

MAEKAWA, R.; CARVALHO, M. M.; OLIVEIRA, O. J. Um estudo sobre a certificação ISO 9001 no Brasil: mapeamento de motivações, benefícios e dificuldades. Gestão \& Produção, v. 20, n.4, p. 763-779, 2013. DOI: https://doi.org/10.1590/S0104-530X2013005000003

MAGANHA, M. R. J.; RODRIGUES, J. S.; LEONI, J. N. Diagnóstico do nível de maturidade do processo de desenvolvimento de produtos: um estudo de caso em empresa calçadista.

Gestão da Produção, Operações e Sistemas, v. 9, n.1, p. 57-67, 2014.

DOI: https://doi.org/10.15675/gepros.v0i1.1141 
MELLO, L. T. C.; DORNFELD, H. C. M.; GUILHERME DOS SANTOS, G.; PASSOS, D.; RIBEIRO, R.; GODINHO FILHO, M. Análise do lead time nos processo logísticos de uma rede varejista de flores. Produção Online, v. 16, n.4, p. 1237-1261, 2016.

DOI: https://doi.org/10.14488/1676-1901.v16i4.2253

MENDES, G. H. S.; TOLEDO, J. C. Explorando práticas do desenvolvimento de produtos em pequenas e médias empresas do setor de equipamentos médico-hospitalares. Gestão \& Produção, v. 19, n.1, p. 103-117, 2012.

DOI: https://doi.org/10.1590/S0104-530X2012000100008

NORONHA, J. C.; MELLO, C. H. P.; SANCHES DA SILVA, C. E.; NORONHA, J. C. C. Opções reais aplicadas à gestão do processo de desenvolvimento de produtos em uma indústria de autopeças. Gestão \& Produção, v. 21, n.1, p. 77-94, 2014.

DOI: https://doi.org/10.1590/S0104-530X2014000100006

PAIVA, C. H. A. A Burocracia no Brasil: as bases da administração pública nacional em perspectiva histórica (1920-1945). História, v. 28, n.2, p. 775-796, 2009.

DOI: https://doi.org/10.1590/S0101-90742009000200027

PEREIRA SOUZA, L.; FARIA NETO, A.; MUNIZ JÚNIOR, J. Análise crítica do processo de auditoria de sistema de gestão da qualidade no setor aeroespacial. Gestão \& Produção, v. 19, n.1, p. 31-41, 2012. DOI: https://doi.org/10.1590/S0104-530X2012000100003

QUINTELLA, H. L. M. M.; ROCHA, H. M. Nível de maturidade e comparação dos PDPs de produtos automotivos. Produção, v. 17, n. 1, p. 199-217, 2007.

DOI: https://doi.org/10.1590/S0103-65132007000100014

RODRIGUES, C. H. R.; ALMADA SANTOS, F. C. Empowerment: ciclo de implementação, dimensões e tipologia. Gestão \& Produção, v. 8, n.3, p. 3237-249, 2001.

DOI: https://doi.org/10.1590/S0104-530X2001000300003

SAES, E. V.; GODINHO FILHO, M. Utilização da abordagem Quick Response Manufacturing em uma empresa de materiais de escrita: proposta e análise de benefícios esperados.

Gestão \& Produção, v. 18, n.1, p. 525-540, 2011.

DOI: https://doi.org/10.1590/S0104-530X2011000300007

SAPKAUSKIENE, A.; LEITONIENE, S. The concept of Time-Based Competition in the context of Management Theory. Engineering Economics, v.21, n.2, p.205-2013, 2010.

SARAIVA, L. A. S. Cultura Organizacional em Ambiente Burocrático. Revista de

Administração Contemporânea, v. 6, n.1, p. 187-207, 2002.

DOI: https://doi.org/10.1590/S1415-65552002000100011

SOULÉ, F. V.; FRANÇOSO, A. B. L.; COSTA, L. B. M.; KODAMA, T. K.; BESSI, N. C.; SCHIAVON, L.; GODINHO FILHO, M. Proposta de redução de lead time na linha de produtos termoelétricos de uma empresa familiar no interior paulista. Produção Online, v. 16, n. 1, p. 278-312, 2016. DOI: https://doi.org/10.14488/1676-1901.v16i1.2138

STALK JR., G. J. Time: the next source of competitive advantage. Harvard Business Review, 66, July-August, 44-51, 1988. 
SUN, H.; ZHAO, Y.; YAU, H. K. The relationship between quality management and the speed of new product development. The TQM Journal, v. 21, n.6, p. 576-588, 2009. DOI: https://doi.org/10.1108/17542730910995855

SURI, R. Quick Response manufacturing: a companywide approach to reducing lead times. Portland, Oregon: Portland, 1998.

SURI, R. It's about time - The competitive advantage of Quick Response

Manufacturing. New York: Productivity Press, 2010.

DOI:https://doi.org/10.1201/EBK1439805954

TYAGI, S.; CHOUDHARY, A.; CAI, X.; YANG, K. Value stream mapping to reduce the leadtime of a product development process. International Journal Production Economics, v. 160, p. 202-212, 2015. DOI: https://doi.org/10.1016/.ijpe.2014.11.002

TOLEDO, J. C.; LULS DA SILVA, S.; ALLIPANDRINI, D. H.; MARTINS, M. F.; FERRARI, F. M. Práticas de gestão no desenvolvimento de produtos em empresas de autopeças.

Produção, v. 18, n.2, p. 405-422, 2008.

DOI: https://doi.org/10.1590/S0103-65132008000200015

TOLEDO, J. C.; SIMÕES, J. M. S. Gestão do desenvolvimento de produto em empresas de pequeno e médio porte do setor de máquinas e implementos agrícolas do Estado de SP.

Gestão \& Produção, v. 17, n. 2, p. 257-269, 2010.

DOI: https://doi.org/10.1590/S0104-530X2010000200004

VEERAMANI, D.; AGRAWAL, R. K. Living systems theory based modeling for reengineering office operations. Journalof Manufacturing Systems, v. 18, n. 5, p. 323-334, 1999.

DOI: https://doi.org/10.1016/S0278-6125(00)87635-9

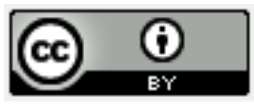

Artigo recebido em 20/04/2017 e aceito para publicação em 01/06/2017

DOI: http://dx.doi.org/10.14488/1676-1901.v18i2.2844 